\title{
An Intelligent Logistics Tracking System Based on Wireless Sensor Network
}

\author{
https://doi.org/10.3991/ijoe.v14i01.8063 \\ Jijun Xing \\ Northwest University of Political Science and Law, Xi'an, Shaanxi, China \\ lfwv82271723xu@163.com
}

\begin{abstract}
In view of the shortcomings in information collection and tracking management in existing logistics, the key technologies of wireless sensor network and the basic concepts and methods of Information Technology Infrastructure Library(ITIL) is studied. The basic structure and main functions of the logistics tracking system based on wireless sensor network is put forward. It can effectively solve the detection, location, recognition and automatic processing of fault events and other issues of goods in the process of logistics tracking management. In addition, combined with the GIS software, the logistics tracking and monitoring system based on the wireless sensor network is realized by using Java language coding. The results show that this system can realize the tracking, visualization and automatic management of the information in the process of goods transportation. Therefore, the proposed method significantly improves the accuracy, rapidity and intuition of the logistics information management.
\end{abstract}

Keywords-wireless sensor networks, logistics tracking, monitoring system, ITIL

\section{Introduction}

At present, with the rapidly development of economic globalization and information technology, the service economy has developed rapidly in the world, and the logistics industry as a new service sector has been rising rapidly in the world [1]. More and more abundant materials bring convenience to people's lives, but also bring some impact. How to store and transport the large number of goods with various kinds for effective information management has become an important issue. In the process of information management, it needs to track and trace the materials, and can automatically deal with the fault events. The traditional management methods are no longer applicable. The emergence of wireless sensor network provides the methods and ideas for solving these problems. wireless sensor network can connect any items to the Internet through RFID, WSN and other information sensing equipment. It can achieve information exchange through the network, and is provided with the fast acquisition speed and high degree of automation. The logistics tracking management system based on wireless sensor network can realize the whole process visual control 
of purchasing, transportation, storage, distribution and use in the logistics supply chain, and can automatically deal with the fault events and promote the logistics management information [2].

Because of the time-sensitive nature of the perishable food, it is required that the cold chain be able to perform the real-time low-temperature environmental monitoring function and keep the cold chain's low-temperature transportation environment, therefore keeping the low temperature environment is the core requirement of the cold chain system. If the temperature control is not accurate enough, it will lead to a series of low-quality products [3]. Each link of cold chain, from the picking of the product to being sold out, need to participate in control. Every link in the chain can go wrong and break the cold chain, either on the platform of a warehouse, in transit, in storage, or in a retail supermarket, which can affect the final consumer's demand. At present, China's cold chain monitoring system is relatively sound, and sound monitoring measures have been taken in the production, storage and sales. But the cold chain monitoring in the transport is relatively weak. Cold chain system based on wireless sensor network makes sure that the food is transported in the specified lowtemperature environment to keep the original freshness, color and nutrition of the food and ensure the food quality and safety [4].

\section{State of the art}

Wireless sensor network is a series of independent systems built on the part of infrastructure of existing Internet. It has a unique intelligent infrastructure. Radio Frequency Identification (RFID) is one of the key technologies of wireless sensor network. The wireless sensor network can collect information fast and accurately with RFID technology, and make the information interconnect with the traditional Internet [5]. Communication modes of wireless sensor network includes things and things communication, things and people communication, people and people communication and other different types, which makes the virtual network world extend to real life. The practical application of the wireless sensor network provides a new opportunity for the development of the logistics industry, gives the goods in the process of transport intelligence, and turns the logistics information management into intelligent. Instrumented, networking, perception, automation and intelligence are the basic characteristics of things [6].

The intelligent, location, tracking, monitoring and management of the wireless sensor network is embodied in the field of logistics as a centralized data processing and service center based on the network. It uses radio frequency identification devises (RFID) to collect material information, and transmits information to the data service center through the network. It also integrates the material information on the map displayed by GIS system to achieve the visibility control of material [7-8].

In foreign countries, the intelligent logistics starts some earlier. The development of intelligent logistics of some countries in Europe and the United States is in a leading position. In the United States, the federal logistics company developed a set of logistics system based on wireless sensor network for real-time monitoring of logis- 
tics. The system calculates the data uploaded through the sensor in the server-side, if the logistics has problems, it can alarm in time. And the user can monitor the dynamic of the goods in time, which is easy to transport some dangerous goods, fragile goods and high value goods. Caterpillar has developed a system which is specifically designed for intelligent logistics. The system can simulate, plan, evaluate and forecast the process of warehousing and freight transportation. In Japan, the intelligent logistics develops rapidly, and the intelligent logistics is applied in the logistics transportation of cold chain, fresh chain and chemical industry [9].

In the country, the national policy attaches importance to wireless sensor network, and more and more technology companies want to develop on the intelligent logistics. The development of intelligent logistics of some traffic hub cities in China is much higher than other cities. Major electronic business develops rapidly in the field of ecommerce, but also aware the importance of the logistics industry in the supply chain, have increased logistics construction, and Jingdong, Suning and other electricity providers continue to invest in logistics warehousing. With the rapidly development of upstream and downstream industries of supply chain, the logistics industry has also been paid more and more attention [10]. At the same time, the arrival of the era of large data brings opportunities and challenges to the development of intelligent logistics. The storage, processing, query of logistics information uploaded all the time makes the traditional data storage become powerless. Therefore, the new logistics information system of wireless sensor network should improve the storage way of data.

\section{$3 \quad$ Methodology}

\subsection{The overall structure of system}

The system is mainly divided into server layer and application layer. The server layer is the bottom of the application layer. Server layer will deal with the distribution of goods, invoicing and inventory information collected through a variety of sensors [11]. Application layer mainly turns the information processed by server layer into events, and stores the information in the database in accordance with the event format required by logistics enterprises. And then the different information is showed to each user in the system interface according to the different user rights to query and maintain conveniently. The overall structure of logistics tracking system based on wireless sensor network is shown in Figure 1. 


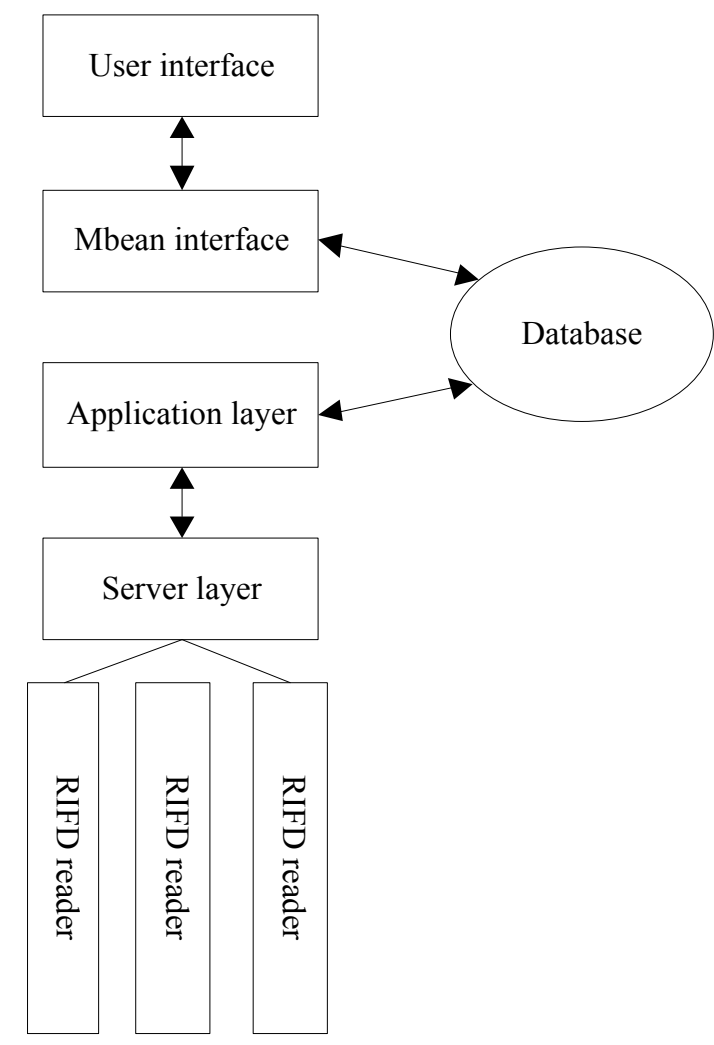

Fig. 1. Overall structure of logistics tracking system based on wireless sensor network

\subsection{System composition}

This paper evaluates the advantages and disadvantages of wireless sensor network coverage optimization algorithm from the following aspects:

Coverage rate: the coverage capability of network is an important index to measure the performance of sleep mechanism. Network coverage is usually used to reflect the coverage capacity of the network. In general, it is defined as the ratio of the effective coverage area Aa of the network to the area A of the target area, that is:

Server layer. Server layer is divided into three layers: sensor abstraction layer, application engine layer and communication layer. The sensor abstraction layer provides a common API that integrates with the LLRP sensor adapter, the Alien sensor adapter, the Borcorde sensor adapter, and the DB sensor adapter to collect various data information from the sensor and generate raw events. The application engine layer filters the collected original events according to event handling rules to get the industrial events needed by logistics ebterprises. Through the TCP and SOAP protocol, JMS and RMI technology, communication layer will use the event of the industry in the engine layer to integrate with other systems (such as: database, operation and maintenance systems, etc.) 
Sensor abstraction layer: server layer connects to Borcorde reader, RFID reader, terminal mobile devices and wireless sensors and other sensors, from which to collect logistics information for processing. The sensor abstraction layer allows the user to collect all the information needed for the application in a transparent manner. Application Engine Layers: The application engine layer processes a large number of redundant events generated by the sensor abstraction layer and generates meaningful events needed by the logistics industry. The server uses a sophisticated event processing engine Esper that filters and identifies valuable events from a large number of event streams according to rules. Esper allows user to write query statements that are similar to database syntax. The overall architecture of the server layer is shown in Figure 2.

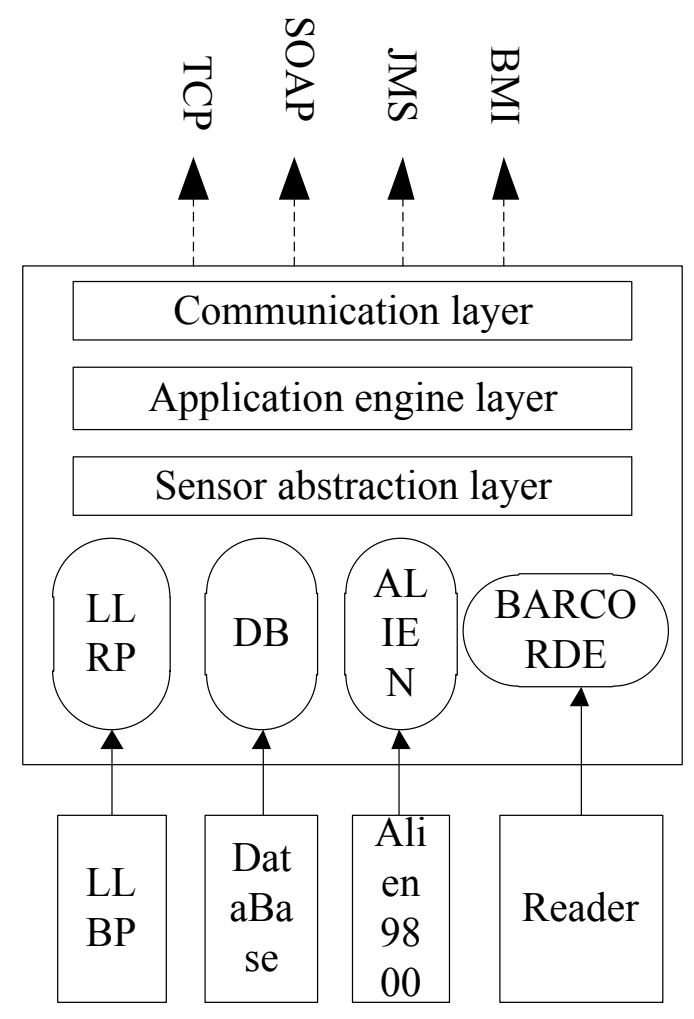

Fig. 2. Overall structure of server layer

Communication layer: the communication layer will submit the processed data to the application system for integration to realize the information exchange. The design of various application interfaces in this layer achieves effective integration with the existing application system.

Application layer. Application layer mainly turns the event information processed by the server layer into the event information needed by the logistics industry, and 
then the event information is stored in the database for the display, viewing and dealing with in user interface. In the distribution process, there are two main events: normal events and abnormal events. The normal events include that the designated items reach to the designated location and leave the designated location. Abnormal events indicate that the items are not transported according to the requirements, which includes that items have missed a place, the goods are shipped back, the items don't follow the prescribed route to transport, and there is no corresponding item name and the corresponding location name. The specific process is shown in Figure 3.

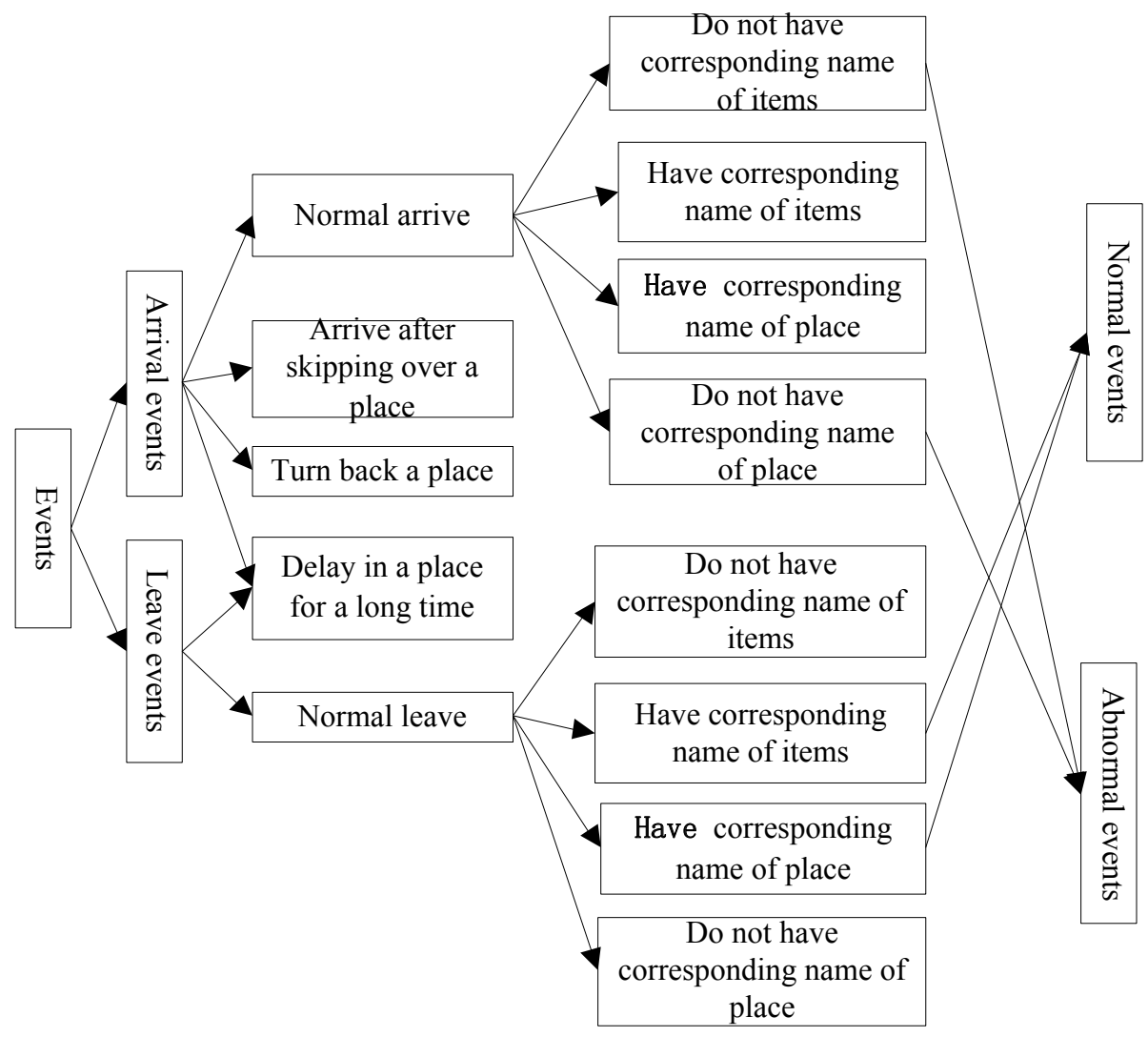

Fig. 3. Application layer events analysis

\subsection{System flow design}

In the logistics business process, the system refers to the best practice and the related standard of the ITIL, and designs the operable, distributed and automatic processing flow according to the event processing in the process of tracking the goods [12]. The design adopts the multi-flow method. The normal events stored in the database by the application layer are handled according to the event work order flow. The abnormal events are processed according to the problem work order flow, and the 
emergency situation such as line adjustment during the transportation of the goods is processed according to the change work order flow. Event work order can be generated automatically or manually by the failure alarm event during work order processing. Event work order can lead to change work orders and problem work order. Users and customers can query the detail information of the transport process of goods through the system's service desk. Maintenance personnel of logistics operation can deal with the events produced in the transport of goods in accordance with the flow, and manage the assets of logistics enterprises.

RFID module uses high-frequency RFID devices to collect data, and the goods in transit can be real-time queried. The user can get the information of the arrived items in time through text messages and Email, and can query the historical information. Geographic information system (GIS) is a comprehensive technical system that collects, stores, computes, retrieves, analyzes, displays and describes the geographical information in the whole or part of the Earth's surface space, supported by the computer network system. The geographic information system has the function of displaying the geographical location information of the articles accurately. The system uses this function and the official Chinese map API interface provided by Google to develop to realize the visualization of the geographical information during the transportation of goods, and can improve the accuracy of the display and maintain the articles line. The event generated by the system is processed through the flow process interface in accordance with pre-designed ITIL process, including the event work order process, the problem work order process and change work order process. The system automatically displays the work order to be processed on the corresponding label card. The system will automatically flow to the corresponding label card of the work order handler of the next task node for the work order that the current node task is processed. In the process of the whole work order, the process of the work order is in the closed-loop state, which greatly improves the efficiency of the work order processing.

\section{$4 \quad$ Result analysis and discussion}

With the continuous development of logistics management theory and the deepening of logistics practice, it is necessary to establish the corresponding logistics performance evaluation method. The corresponding performance evaluation index is determined, to objectively and scientifically reflect the operation of logistics system. Logistics performance evaluation index has its own characteristics, and its content is more extensive than the current enterprise evaluation index. It not only replaces the accounting data, but also puts forward some methods to determine whether the logistics system has the ability to meet the customer or market demand in time. In the actual operation, in order to establish an effective evaluation index system of logistics performance, the following principles should be followed when measuring logistics performance: First, the key performance indicators should be analyzed emphatically. Second, the performance index system should reflect the logistics business process. Third, the index should reflect the operation of the whole logistics system, rather than 
reflecting the operation of the single node enterprise. Fourth, the method of real-time analysis and evaluation should be used as far as possible. Logistics real-time operational information is more valuable than post-mortem analysis. Fifth, performance evaluation indicators can reflect the relationship between suppliers, manufacturers and customers. Sixth, performance evaluation indicators should be able to fully reflect the competitive advantage and competitive ability of logistics.

According to the principle of LSC algorithm, LSC algorithm is a distributed algorithm. It can cluster nodes in the asynchronous state of sensor nodes, and can realize the merging of different subnets. In addition, it has very strong extensibility. Node clustering algorithms that employ large-scale WSNs data collection also need to meet stability requirements. In order to evaluate the clustering stability of sensor nodes, simulation was carried out. The simulation parameters are shown in Table 1.

Figure 4 shows the network size of 2000 sensor nodes. When the communication distance in the cluster is $\mathrm{r}=70 \mathrm{~m}$ and the communication distance between the clusters is $\mathrm{R}=150 \mathrm{~m}$, the number of cluster head nodes and cluster member nodes in 15 clusters is observed. It can be seen that the number of cluster head nodes and cluster member nodes always tends to be stable. The number of cluster head nodes and cluster member nodes in a cluster process is shown in Figure 4.

Table 1. Simulation parameters

\begin{tabular}{|l|l|}
\hline \multicolumn{1}{|c|}{ Parameter name } & \multicolumn{1}{c|}{ Parameter values } \\
\hline Target area & $1000 \times 1000 \mathrm{~m}^{2}$ \\
\hline Number of nodes & $100 \sim 4000$ \\
\hline Node's communication radius & $\begin{array}{l}\text { The communication distance between the clusters is } 150 \mathrm{~m}, \text { and } \\
\text { the communication distance within the cluster is } 70 \mathrm{~m} .\end{array}$ \\
\hline
\end{tabular}

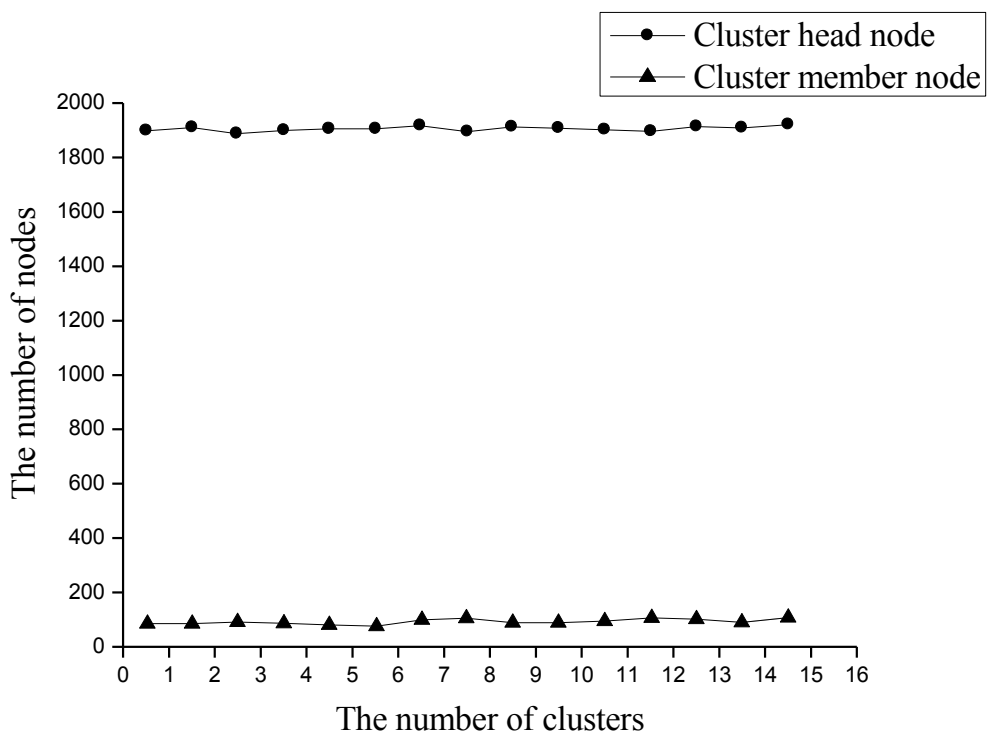

Fig. 4. The number of cluster head nodes and cluster member nodes in a cluster process 
The influence of the number of sensor nodes on clustering is shown in Figure 5.

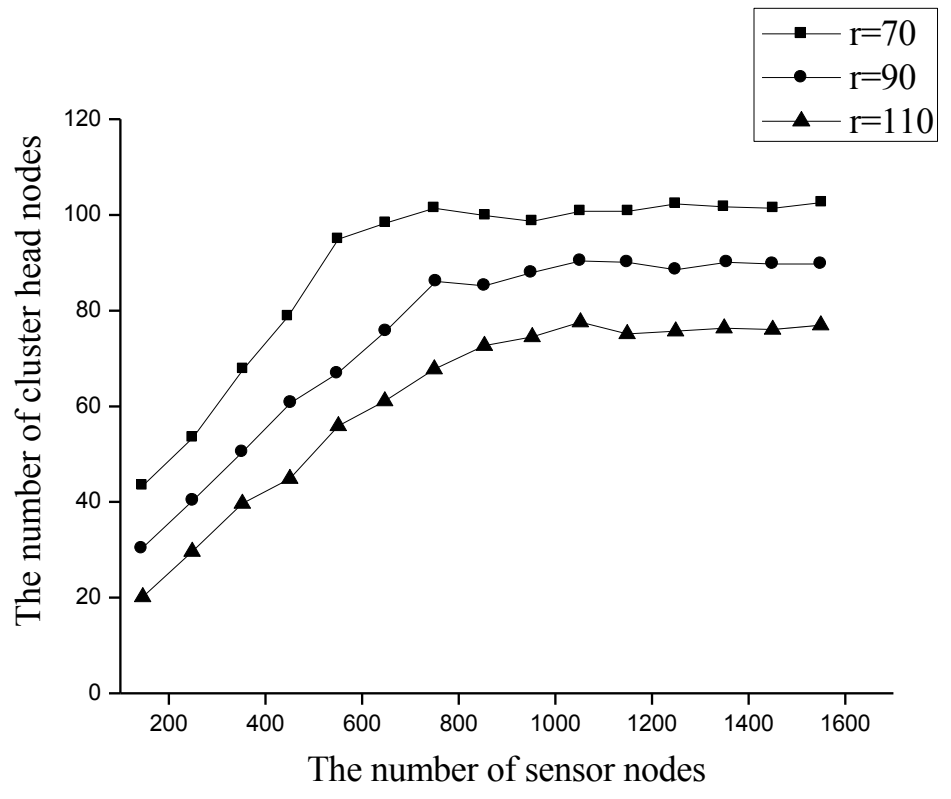

Fig. 5. The influence of the number of sensor nodes on clustering

In the simulation calculation, the number of sensor nodes changes from 100 to 1500 , and the interval increases by 100 . When the number of nodes in the network increased to a certain scale, the number of cluster head nodes maintained at a stable level. The node density, which maintains the level of clustering stability, becomes the critical node density for stable clustering. It is related to the average number of nodes covered by the sensor node communication radius range.

In order to collect the three-dimensional acceleration data of cargo under different behaviors conveniently, the cargo behavior detection system is simplified. The simplified lab consists of 1 carton, 4 3D acceleration sensor test nodes, 1 USB hub and 1 laptop. The test was carried out in the subway car. Four kinds of cargo abnormal behavior were simulated 20 times. Each simulation records the behavior of the goods (balance, shaking, tipping over, moving, impact), time of occurrence and end time. Cargo on the three-dimensional acceleration sensor detection node detection data sent to the computer through the USB interface for storage. Each data received by the monitoring center has GPS information and time information. Using the api interface provided by GoogleMap, we can restore the shipping of a shipment on a GIS map. During the experiment, the corresponding recorded goods behavior are: impact, shaking, moving, moving and tipping over. The test result is consistent with the record result. After examining the 4 types of abnormal behavior data of the goods, the test results are shown in Table 2: 
Paper-An Intelligent Logistics Tracking System Based on Wireless Sensor Network

Table 2. Inspection and verification of abnormal behavior experiment of cargo

\begin{tabular}{|l|c|c|c|c|}
\hline Cargo behavior & Accuracy /\% & Leakage rate /\% & false positive /\% & $\begin{array}{c}\text { Misjudgment } \\
\text { results / sources }\end{array}$ \\
\hline Tipping over & 100 & 0 & 0 & - \\
\hline Impact & 100 & 0 & 0 & - \\
\hline Shaking & 100 & 0 & 3.2 & moving \\
\hline Moving & 97.6 & 2.4 & 5.3 & shaking, balance \\
\hline
\end{tabular}

As can be seen from Table 2, the LSC algorithm can accurately detect the tipping and collision of goods. Based on the maximum similarity of multi-feature recognition, it ensures that goods with obvious characteristics can be preferentially detected. By comparing the recorded results with the test results and observing the characteristics of the three-dimensional acceleration data of the cargo when the fault is detected, it is found that the fuzziness of the abnormal behavior of the cargo leads to a wrong detection result. Among them, a slight and steady movement has not been detected and it is considered that the goods are in a state of equilibrium. The rapid acceleration and shutdown of the train results in a steady increase in the three-dimensional acceleration of the cargo, which is mistakenly detected as the movement of the cargo itself. The slight sloshing of the cargo (small amplitude) will also be detected as moving. From the above analysis, it can be seen that the LSC algorithm has higher accuracy for the detection of the abnormal behavior of goods. In the previous 80 tests, the error rate is less than $5 \%$.

The algorithm can automatically adjust the number of fitting coefficients under the premise of ensuring the accuracy of data acquisition. Therefore, the error can always be controlled within a certain range. The relation between the error and the ratio of the cluster head is shown in Figure 6.

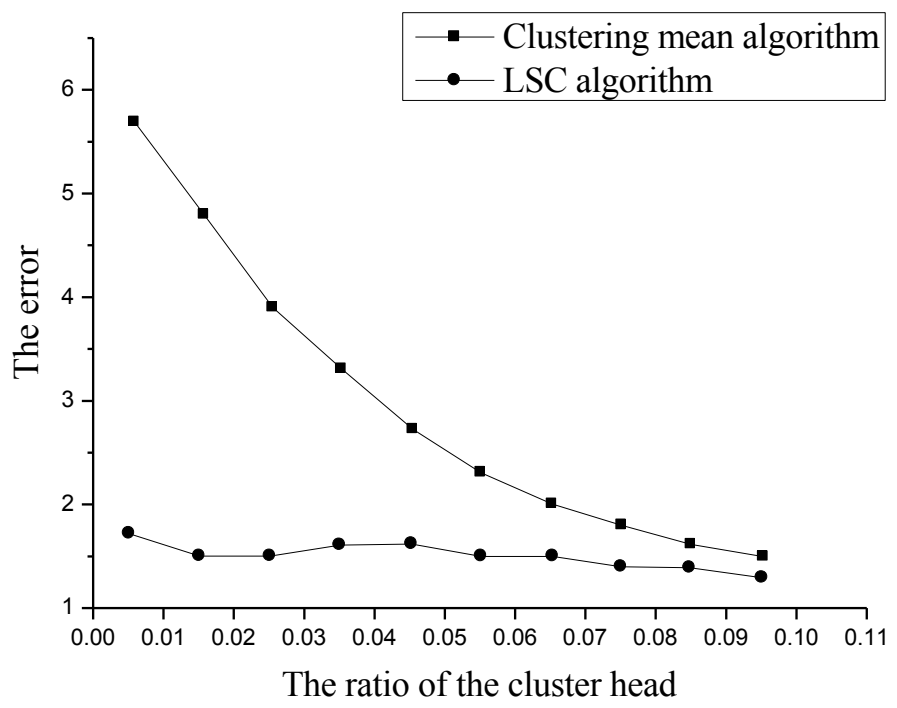

Fig. 6. The relation between the error and the ratio of the cluster head 


\section{Conclusions}

Cargo tracking system is a multidisciplinary complex system engineering. It involves the Internet of things, wireless sensor technology, information technology, data fusion and other disciplines. The application of GPS technology is very important to the location of goods. However, during the transportation of the goods, the measurement information is inaccurate with the change of the surrounding buildings. The logistics tracking management system based on wireless sensor network technology is designed. The system realizes the tracking and monitoring of articles in the process of goods transportation, and adopts the ITIL idea to process the events produced in the process of transportation and realize the automatic management. The realization of the system makes the management of logistics and transportation more intelligent and humanized, and promotes the development of logistics industry information construction. The emergence of wireless sensor network has brought new opportunities for the development of the logistics industry. With the popularity of RFID tags, information technology development of logistics industry will enter a new stage.

\section{References}

[1] Ding, K., Jiang, P., Sun, P., \& Wang, C. (2016). RFID-enabled physical object tracking in process flow based on an enhanced graphical deduction modeling method. IEEE Transactions on Systems, Man, and Cybernetics: Systems.

[2] Fadiya, O., Georgakis, P., Chinyio, E., \& Nwagboso, C. (2015). Decision-making framework for selecting ICT-based construction logistics systems. Journal of Engineering, Design and Technology, 13(2), 260-281.

[3] Huang, P., Xiao, L., Soltani, S., Mutka, M. W., \& Xi, N. (2013). The evolution of MAC protocols in wireless sensor networks: A survey. IEEE communications surveys \& tutorials, 15(1), 101-120. https://doi.org/10.1109/SURV.2012.040412.00105

[4] Hinkka, V., Häkkinen, M., Holmström, J., \& Främling, K. (2015). Supply chain typology for configuring cost-efficient tracking in fashion logistics. The International Journal of Logistics Management, 26(1), 42-60. https://doi.org/10.1108/IJLM-03-2011-0016

[5] Mejjaouli, S., \& Babiceanu, R. F. (2015). RFID-wireless sensor networks integration: Decision models and optimization of logistics systems operations. Journal of Manufacturing Systems, 35, 234-245. https://doi.org/10.1016/j.jmsy.2015.02.005

[6] Oliveira, R. R., Cardoso, I. M., Barbosa, J. L., da Costa, C. A., \& Prado, M. P. (2015). An intelligent model for logistics management based on geofencing algorithms and RFID technology. Expert Systems with Applications, 42(15), 6082-6097. https://doi.org/10.1016/ j.eswa.2015.04.001

[7] Pak, J. M., Ahn, C. K., Shmaliy, Y. S., \& Lim, M. T. (2015). Improving reliability of particle filter-based localization in wireless sensor networks via hybrid particle/FIR filtering. IEEE Transactions on Industrial Informatics, 11(5), 1089-1098. https://doi.org/10.1109/ TII.2015.2462771

[8] Rawat, P., Singh, K. D., Chaouchi, H., \& Bonnin, J. M. (2014). Wireless sensor networks: a survey on recent developments and potential synergies. The Journal of supercomputing, 68(1), 1-48. https://doi.org/10.1007/s11227-013-1021-9 
[9] Shamsuzzoha, A. H. M., Ehrs, M., Addo-Tenkorang, R., Nguyen, D., \& Helo, P. T. (2013). Performance evaluation of tracking and tracing for logistics operations. International Journal of Shipping and Transport Logistics, 5(1), 31-54. https://doi.org/10.1504/ IJSTL.2013.050587

[10] Xu, N., Zhang, Y., Zhang, D., Zhao, S., \& Fu, W. (2017). Moving Target Tracking in Three-Dimensional Space with Wireless Sensor Network. Wireless Personal Communications, 94(4), 3403-3413. https://doi.org/10.1007/s11277-016-3783-x

[11] Yan, Q. (2015). Research on Fresh Produce Food Cold Chain Logistics Tracking System Based on RFID. Adv. J. Food Sci. Technol, 7, 191-194. https://doi.org/10.19026/ajfst. 7.1292

[12] Yu, Q. (2016). Design of Logistics Tracking and Monitoring System Based on Internet of Things. Journal of Residuals Science \& Technology, 13(5).

\section{$7 \quad$ Author}

Jijun Xing is with School of Business, Northwest University of Political Science and Law, Xi'an, Shaanxi, 710063, China.

Article submitted 03 December 2017. Published as resubmitted by the author 10 January 2018. 